\title{
De novo Transcriptome Reveals Gene Changes in the Development of the Endosperm Chalazal Haustorium in Taxillus chinensis (DC.) Danser
}

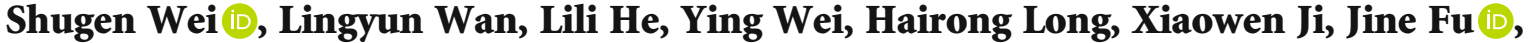 \\ and Limei Pan it \\ Guangxi Botanical Garden of Medicinal Plants, Nanning 530023, China \\ Correspondence should be addressed to Jine Fu; duanwei3014@163.com and Limei Pan; plimei2006@163.com
}

Received 17 August 2019; Accepted 26 December 2019; Published 20 February 2020

Academic Editor: Pengjun Shi

Copyright (c) 2020 Shugen Wei et al. This is an open access article distributed under the Creative Commons Attribution License, which permits unrestricted use, distribution, and reproduction in any medium, provided the original work is properly cited.

Loranthus (Taxillus chinensis) is a facultative, hemiparasite and stem parasitic plant that attacks other plants for living. Transcriptome sequencing and bioinformatics analysis were applied in this study to identify the gene expression profiles of fresh seeds (CK), baby (FB), and adult haustoria tissues (FD). We assembled 160,571 loranthus genes, of which 64,926, 35,417, and 47,249 were aligned to NR, GO, and KEGG pathway databases, respectively. We identified 14,295, 15,921, and 16,402 genes in $\mathrm{CK}, \mathrm{FB}$, and FD, respectively. We next identified 5,480 differentially expressed genes (DEGs) in the process, of which 258, 174, 81, and 94 were encoding ribosomal proteins (RP), transcription factors (TF), ubiquitin, and disease resistance proteins, respectively. Some DEGs were identified to be upregulated along with the haustoria development (e.g., 68 RP and 26 ubiquitin genes). Notably, 36 RP DEGs peak at FB; 10 ER, 5 WRKY, 6 bHLH, and 4 MYB TF genes upregulated only in FD. Further, we identified 4 out of 32 microRNA genes dysregulated in the loranthus haustoria development. This is the first haustoria transcriptome of loranthus, and our findings will improve our understanding of the molecular mechanism of haustoria.

\section{Introduction}

Taxillus chinensis (DC.) Danser, also called loranthus or "San Ji Sheng" (in Chinese), is a member of Loranthaceae family and mainly distributed in the southern and southwestern areas of China. It has a long history of being used in the Chinese traditional medicine, mainly because its stems and leaves can be used for the treatment of rheumatoid arthralgia, threat of abortion, and hypertension [1,2]. Loranthus is a parasitic plant that attacks other plants, such as Aceraceae, Anacardiaceae, Euphorbiaceae, Fabaceae, Fagaceae, Juglandaceae, Moraceae, Rosaceae, and Rutaceae [2]. The successful parasitism is a key process for the plants to obtain water and nutrients from the host plants via specialized feeding structures called haustoria.

In plants, approximately 4,500 parasitic species belonging to 28 families, representing $1 \%$ of the dicotyledonous angiosperm species, have been reported [3]. Depending on the attachment site in the host plants, parasitic plants can be classified in to two groups-stem and root parasites. Also, according to the degree of host dependency, parasites can be facultative or obligate. Facultative parasites can live autotrophically but latter cannot, such as Triphysaria spp. and Phtheirospermum spp., while obligate parasites have to parasitize a host in order to complete their life cycles, for example, Viscum spp., Cuscuta spp., Orobanche spp., and Striga spp.. Further, parasitic plants can be classified as hemiparasites or holoparasites based on whether they have retained or completely lost the photosynthetic activity [3]. Based on these characteristics, loranthus is a facultative, hemiparasite, and stem parasite.

It has been reported that after seed germination, most parasitic plants will develop a functional haustoria depending on a second chemical signal also derived from the host exudate, such as 2,6-dimethoxy-p-benzoquinone (DMBQ), phenolic acids, and flavonoids (a haustoria-inducing factors (HIFs)) [4]. Some studies have shown the mechanisms of haustoria development in parasitic plants. For example, a 
single-electron reducing quinone oxidoreductase (TvPirin) is required to trigger the haustoria development in the roots of Triphysaria versicolor [5]. The seeds of Santalum album, an aggressive root hemiparasite, can germinate in sand or in vitro on Murashige and Skoog medium after a pretreatment of $2 \sim 8 \mathrm{mM} \mathrm{GA}_{3}$ for $12 \mathrm{~h}$ and then develop the haustoria within one month without the need for induction by HIFs [6-8]. Many is unknown about haustoria development in loranthus.

Transcriptome sequencing has been used to identify differentially expressed genes in the process of parasitism of Cuscuta pentagona, including genes encoding plant hormone (e.g., auxin, gibberellin, and strigolactones), transporters, and genes associated with cell wall modifications [9]. Also, it has been used to show that genes involved in cell wall metabolism, protein metabolism, and mitochondrial electron transport, genes related to auxin signaling and genes encoding nodulin-like proteins, were important for the haustoria development in Santalum album [4]. Transcriptome analysis also found that genes related to protein turnover, detoxification of reactive oxygen species, and fungal pathogenesis are abundant in the haustoria of Golovinomyces orontii [10]. Recently, small RNA sequencing characterized that some dodders' (Cuscuta spp.) microRNAs (miRNAs) could target the host (Arabidopsis thaliana) genes and further improve the parasitism [11].

In the present study, we constructed a transcriptome profile of haustoria development and identified genes encoding ribosomal proteins (RPs), transcription factors (TFs), ubiquitin, and disease-resistant proteins (DRPs) which might be involved in the loranthus haustoria development. Our results provide a valuable resource for further exploration and a basis towards understanding the molecular mechanisms of the haustoria development and underlying host-parasite interaction in angiosperms.

\section{Materials and Methods}

2.1. Plant Material. Fifty seeds of Taxillus chinensis (DC.) Danser were collected from the experimental field of Guangxi Botanical Garden of Medicinal Plants in China, confirmed by senior botanists and deposited in the herbarium of Guangxi Botanical Garden of Medical Plants (accession number: s0001794). Then, the seeds were peeled, washed with sterile water, placed on a germination dish, and incubated under the environment of $25^{\circ} \mathrm{C}$ temperature and $80 \%$ moisture. Every day, the seeds were lighted under $2000 \mathrm{Lx}$ for $10 \mathrm{~h}$. Three fresh seeds were collected as control (CK). After 10 days of incubation, three seeds with protruding seed-type radicle and tiny suction device were randomly collected (FB). After 20 days of incubation, the loranthus haustoria was formed and elongated, and the true leaves began to grow. Three of them were collected as adult haustoria (FD).

\subsection{Total RNA Extraction and Transcriptome Sequencing.} Total RNA was extracted using TRIzol reagent, as described $[1,12]$. After the quality and quantity were determined by Agilent 2100 Bioanalyzer, total RNA $(1 \mu \mathrm{g})$ of each sample was used to construct the cDNA library using the TruSeq
RNA Library Preparation Kit v2 protocol (Illumina), as described [13]. Then, cDNA libraries were quality controlled by the Agilent 2100 Bioanalyzer and qRT-PCR, followed by sequencing on the Illumina HiSeq2500 platform with paired-end 100 strategy.

2.3. De Novo of the Transcriptome. Raw data were cleaned using trim_galore (v0.5.0) and quality controlled using FASTQC (v0.11.7). Next, we used Trinity (v2.8.4) to de novo assemble the loranthus haustoria transcriptome with default parameters, as previously described [1].

2.4. Transcriptome Annotation. After the likely proteins were extracted from the assembled transcriptome using TransDecoder, they were annotated using Trinotate (v3.1.1). In this step, likely proteins were searched against the UniProtKB/Swiss-Prot database to identify known proteins, functional PFAM domains were identified using HMMER [14], signal peptides were predicted using SignalP [15], transmembrane domains were predicted using TMHMM Sever v2.0 [16], and rRNA transcripts were predicted using RNAMMER [17]. Then, EggNOG database (v4.1) [18] was searched against to identify proteins in EuKaryotic Orthologous Groups (KOG), Clusters of Orthologous Groups (COGs), and nonsupervised orthologous groups (NOGs).

Next, we annotated the assembled loranthus genes using KEGG pathway Gene Ontology (GO) databases. BLAST software was used to map the assembled genes to the NR database and the hits with and $e$-value of $>1 \times 10^{-5}$ were filtered. Remaining genes were processed to retrieve GO annotation in terms of biological process, cellular component, and molecular function by BLAST2GO [19]. Using the enzyme commission numbers produced by BLAST2GO, we mapped the assembled transcriptome to KEGG pathway database and obtained the pathway annotation.

2.5. Noncoding Gene and miRNA Annotation. Unannotated loranthus genes were processed by the Coding Potential Calculator (CPC, v2) with default parameters to identify potential long noncoding genes [20]. Then, all the plant mature microRNAs (miRNAs) were mapped to these noncoding genes to identify loranthus miRNAs using SOAP2 with maximal two mismatches [21]. Then, MIREAP was used to predict the miRNA precursor sequences, and psRobot was used to predict the target genes of miRNAs [22].

2.6. Gene Expression Profile and Differential Expression Analysis. Bowtie2 and RSEM tools were used to align clean reads to the assembled transcriptome and to profile the gene expression for each sample, respectively, [23]. Transcriptsper-million (TPM) reads method was for normalization, and lowly expressed genes $(\mathrm{TPM}<5)$ were filtered. Then, differential expressed genes (DEGs) were identified using edgeR [24] with a strict criteria: $\log 2$ fold change (Log2FC) $>$ 1 or $<-1$ and false discovery rate (FDR) of $<0.05$.

2.7. Functional Analysis. $p$ value calculated using Fisher's exact test and $q$ value calculated by the R package "qvalue" were used to identify enriched GO terms and KEGG 


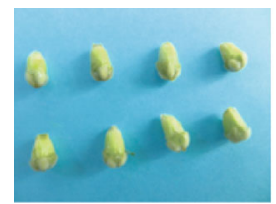

CK

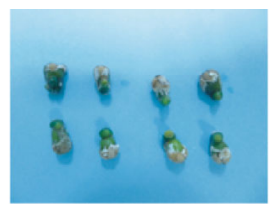

FB

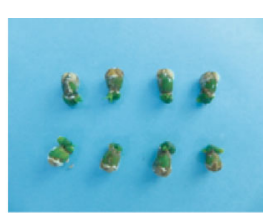

FD

(a)

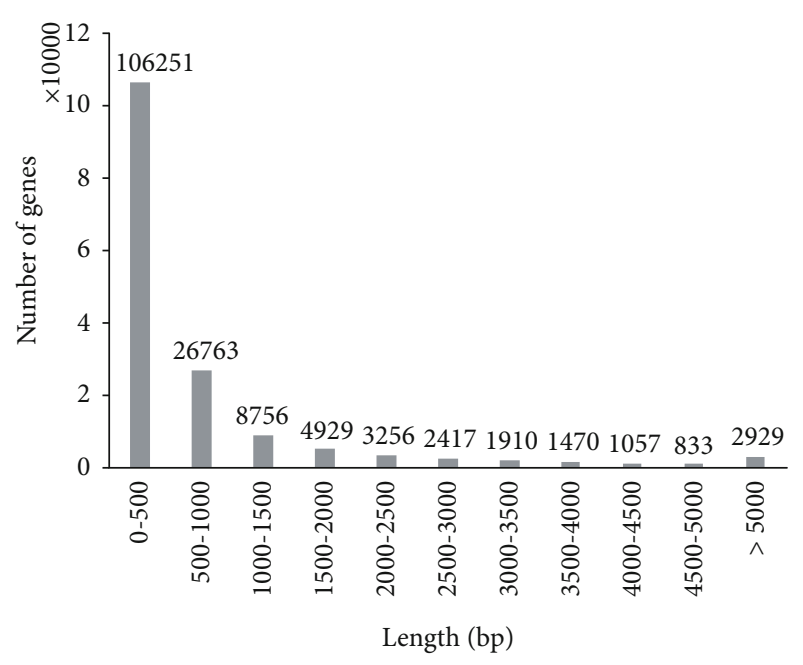

(b)

FIGURE 1: Loranthus seeds and Trinity de novo analysis. (a) Image captures of seeds in three conditions: fresh (CK), baby haustoria (FB), and adult haustoria (FD). (b) Length distribution of assembled loranthus haustoria genes.

TABLE 1: Overview of the transcriptome sequencing and de novo analysis.

\begin{tabular}{|c|c|c|c|c|c|c|c|c|c|}
\hline Sample & CK_R1 & CK_R2 & CK_R3 & FB_R1 & FB_R2 & FB_R3 & FD_R1 & FD_R2 & FD_R3 \\
\hline Raw_reads & 24753420 & 43182897 & 15529733 & 34554423 & 50662597 & 32924448 & 42843339 & 44541750 & 33923755 \\
\hline Clean_reads & 24663725 & 43043674 & 15411513 & 34379375 & 50514766 & 32915342 & 42671084 & 44404033 & 33913639 \\
\hline Genes & & & & & 160571 & & & & \\
\hline Transcripts & & & & & 266379 & & & & \\
\hline GC (\%) & & & & & 42.83 & & & & \\
\hline N50 (bp) & & & & & 1191 & & & & \\
\hline Average_gene length (bp) & & & & & 685.36 & & & & \\
\hline Expressed_genes & 13303 & 13428 & 13538 & 15307 & 15047 & 15087 & 15893 & 15564 & 15587 \\
\hline
\end{tabular}

pathways ( $p$ value of $<0.05$ and $q$ value of $<0.05$ ). Human or other animal-related GO terms and pathways were filtered.

2.8. $q R T-P C R$. We randomly selected 9 genes for $\mathrm{qRT}-\mathrm{PCR}$ validation, and $18 \mathrm{~S}$ rRNA was used as internal control. Forward and reverse primers were predicted using Primer3 and synthesized at BGI-Shenzhen. The procedure of qRTPCR experiment was same as our previous study [1]. The expression of genes was shown in $\Delta \mathrm{Ct} . \Delta \Delta \mathrm{Ct}$ was used to present the difference of gene expression between two samples. Then, we used relative normalized expression (RNE) to show the gene expression changes: $R N E=2^{-\Delta \Delta \mathrm{Ct}} \cdot p$ values were calculated using the multiple $t$ tests function in Prism GraphPad 8.0.

\section{Results}

3.1. Plants, Sequencing, and De Novo Analysis. Compared to $\mathrm{CK}$, the green colors of $\mathrm{FB}$ and FD seeds were darker (Figure 1(a)). In addition, FB seeds produced seed-type radicle and tiny suction device. FD seeds formed and elongated the haustoria, and their true leaves began to grow. We generated a total of $\sim 322.92$ million reads (average: $\sim 35.88$ million reads) for these samples. After data cleaning, $~ 321.92$ million reads were obtained, and Trinity assembled 160,571 loranthus genes that can produce 266,379 transcripts (Table 1). The size of the loranthus haustoria transcriptome was $\sim 110 \mathrm{Mb}$, the GC percentage was $42.83 \%$, the N50 was $1,191 \mathrm{bp}$, which revealed that $50 \%$ of the assembled loranthus genes were $>1,191 \mathrm{bp}$, and the average gene length was $685.36 \mathrm{bp}$. Furthermore, gene length distribution (Figure 1(b)) showed 106,251 (66.17\%) genes between $200 \mathrm{bp}$ to $500 \mathrm{bp}$ and 8,199 (5.11\%) genes longer than $3000 \mathrm{bp}$.

3.2. Annotation of Coding and Noncoding Genes. We next aligned the assembled genes to public databases, including NCBI nonredundant (NR), UniProt/SwissProt, GO, and KEGG pathway (Figure 2(a)). It was shown that the 64,926 genes aligned to NR and that the top five species that are aligned by loranthus genes were Vitis vinifera (grape, 34,147 transcripts), Theobroma cacao (cacao tree, 5,456 transcripts), Nelumbo nucifera (lotus, 5,323 transcripts), Ziziphus jujube (jujube, 4,757 transcripts), and Citrus sinensis (orange, 3,967 transcripts) (Figure 2(b)). Top two GO terms involved by the assembled genes were "metabolic process" $(23,049$ genes) and "cellular process" (22,002 genes) (Figure 2(c)) while the top KEGG pathway involved with the loranthus 


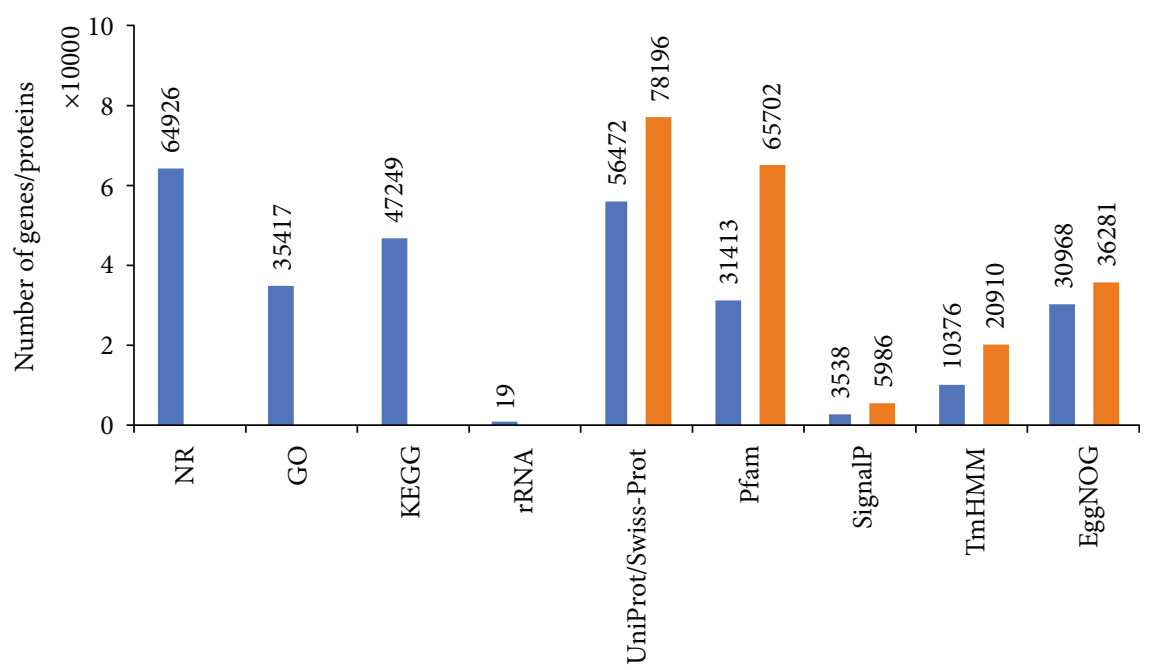

- Gene

- Protein

(a)

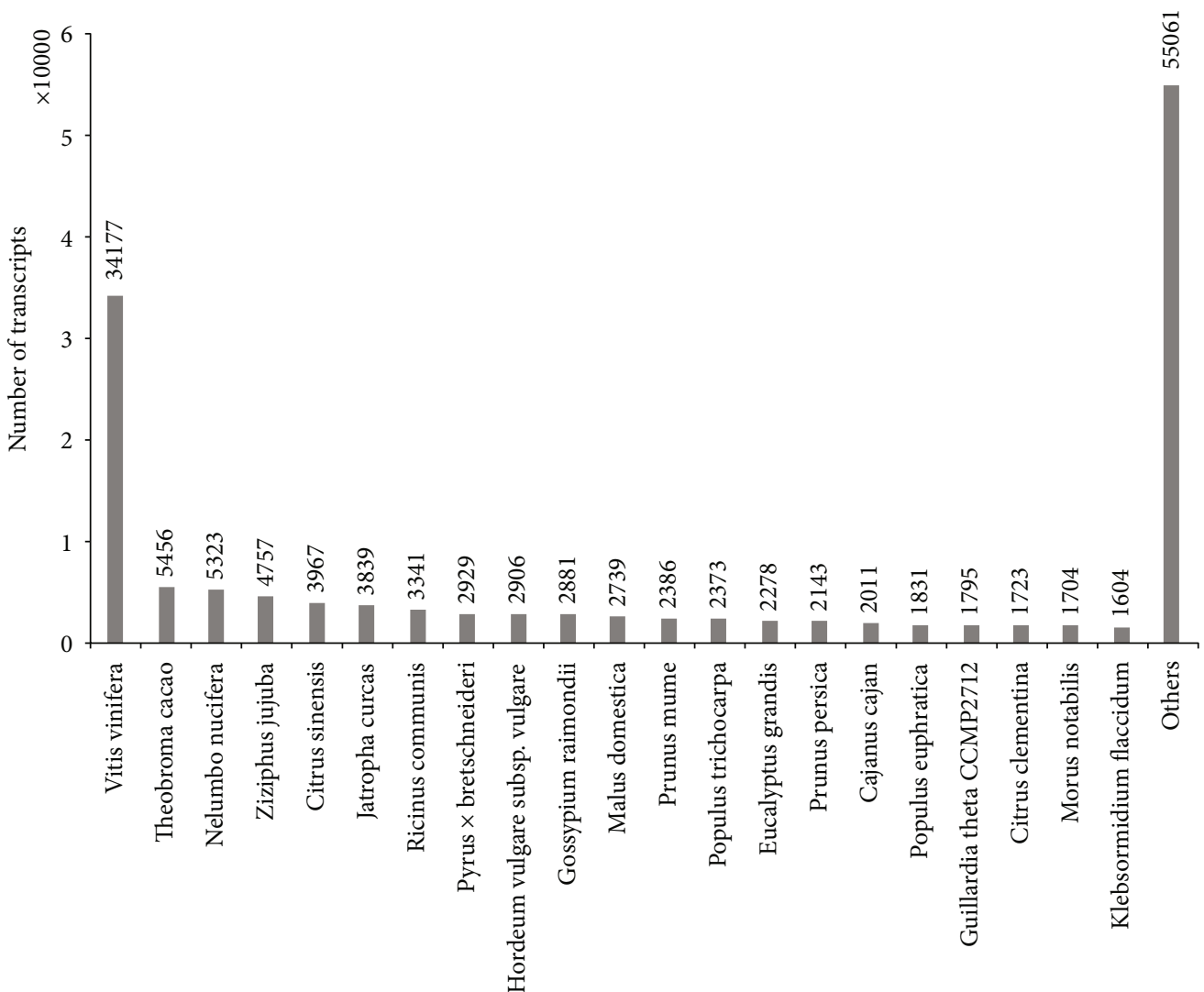

(b)

FIgURe 2: Continued. 


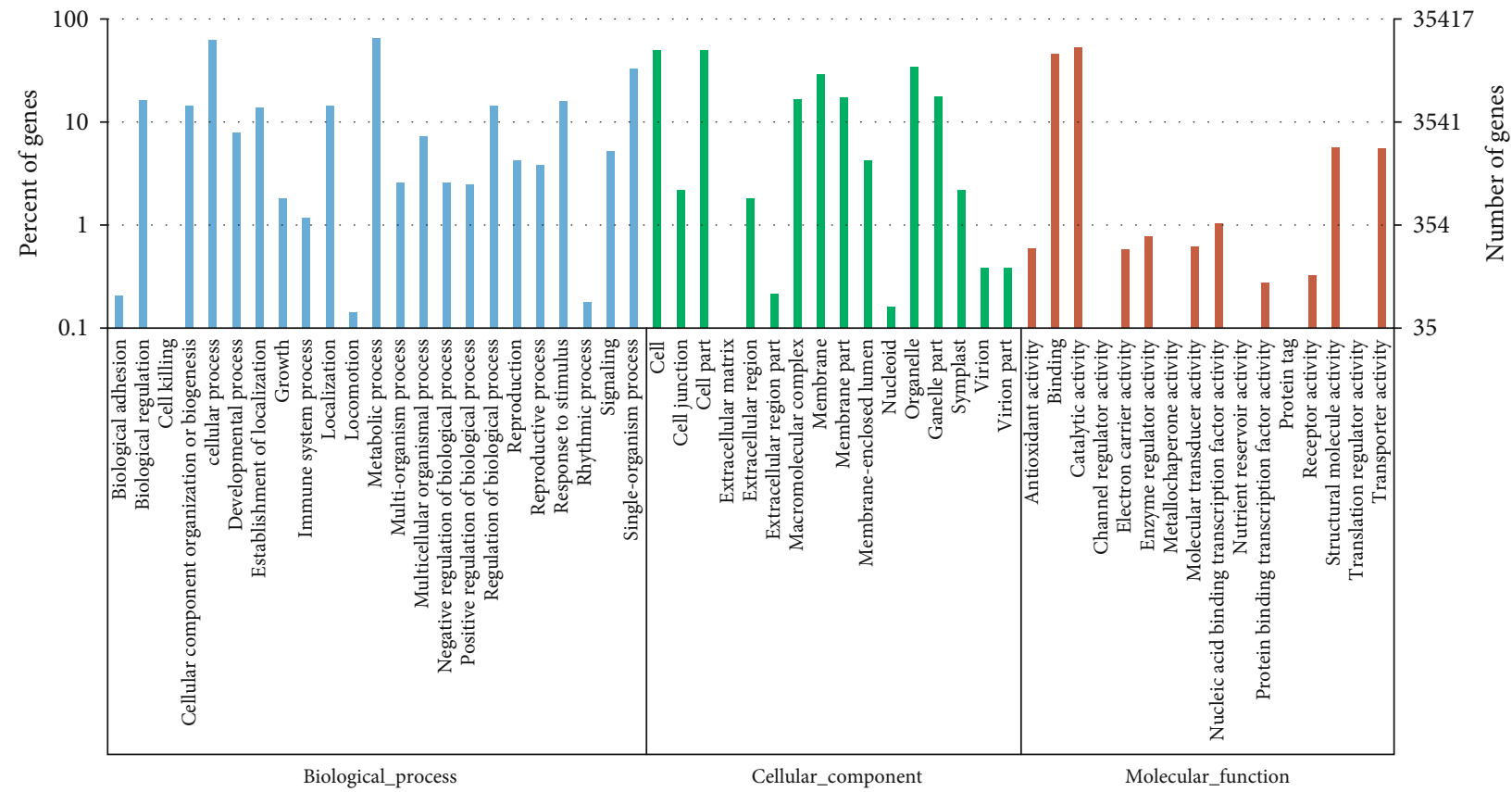

(c)

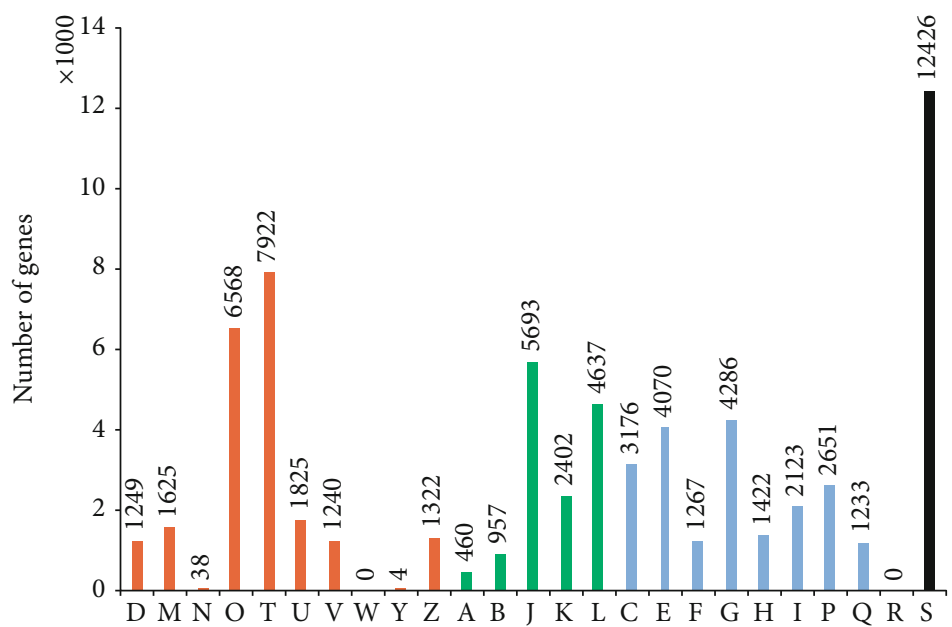

Cellular processes and signaling

D Cell cycle control, cell division, chromosome partitioning M Cell wall/membrane/envelope biogenesis

$\mathrm{N}$ Cell motility

O Posttranslational modification, protein turnover, and chaperones

T Signal transduction mechanisms

$\mathrm{U}$ Intracellular trafficking, secretion, and vesicular transport

$\mathrm{V}$ Defense mechanisms

W Extracellular structures

Y Nuclear structure

Z Cytoskeleton

Information storage and processing

A RNA processing and modification

B Chromatin structure and dynamics

J Translation, ribosomal structure and biogenesis

K Transcription

L Replication, recombination, and repair

Metabolism

C Energy production and conversion

E Amino acid transport and metabolism

F Nucleotide transport and metabolism

G Carbohydrate transport and metabolism

$\mathrm{H}$ Coenzyme transport and metabolism

I Lipid transport and metabolism

$\mathrm{P}$ Inorganic ion transport and metabolism

Q Secondary metabolites biosynthesis, transport, and catabolism

Poorly characterized

$\begin{array}{ll}\mathrm{R} & \text { General function prediction only } \\ \mathrm{S} & \text { Function unknown }\end{array}$

(d)

FIgURE 2: Annotation of the assembled transcriptome. (a) Number of genes aligned to databases. (b) Number of genes aligned to different species. (c) GO annotation of the assembled transcriptome. (d) COG annotation.

genes was "metabolic pathway" (ko01100, 11,714 genes). Notably, we found 1,757 genes related to the pathway of "plant-pathogen interaction" (ko04626).

Then, TransDecoder predicted 96,665 proteins encoded by the loranthus genes and 78,196 (derived from 56,472 genes) were aligned to UniProtKB/SwiiProt database (Figure 2(a)). Next, we identified 65,702 functional Pfam domains, 5,986 signal peptides, and 20,910 transmembrane regions in the likely proteins using HMMER, SignalP, and
TMHMM, respectively (Figure 2(a)). Next, we aligned the loranthus genes to EggNOG database and found that the top three categories were "signal transduction mechanisms" (7,922 genes), "post-translational modification, protein turnover, and chaperones" (6,568 genes), and "translation, ribosomal structure and biogenesis" (5,693 genes) (Figure 2(d)).

RNAMMER predicted 19 genes that can produce ribosomal RNAs in the assembled genes. Next, CPC identified 99,817 potential long noncoding genes in the unannotated 
TABLE 2: Number of DEGs from different families identified in this study.

\begin{tabular}{lccc}
\hline Gene_family & FB_vs_CK & FD_vs_CK & FD_vs_FB \\
\hline Ribosomal protein & $123 / 7$ & $200 / 5$ & $184 / 38$ \\
TF & $80 / 46$ & $101 / 41$ & $32 / 12$ \\
$\quad$ TF_bHLH & $15 / 4$ & $17 / 2$ & $1 / 3$ \\
$\quad$ TF_ER & $9 / 10$ & $18 / 7$ & $13 / 1$ \\
$\quad$ TF_MYB & $7 / 7$ & $12 / 8$ & $5 / 2$ \\
TF_WRKY & $10 / 5$ & $11 / 3$ & $5 / 1$ \\
Ubiquitin & $38 / 17$ & $48 / 11$ & $27 / 15$ \\
Disease-resistant protein & $71 / 6$ & $67 / 1$ & $7 / 8$ \\
\hline
\end{tabular}

genes, of which 32 were predicted to encode microRNAs from 19 microRNA families (Supplementary Table S1). Interestingly, we found that 3,457 protein coding genes might be specific to loranthus according to the CPC label.

3.3. Gene Expression Profile and Differential Expression Analysis. After lowly expressed genes (TPM <5) were filtered, we identified 14,295, 15,921, and 16,402 genes in $\mathrm{CK}, \mathrm{FB}$, and FD, respectively, and found 12,888 genes commonly detected in all three samples. Next, we performed DEG analysis to identify genes involved in the loranthus haustoria development. Using edgeR, we identified 3,749 and 4,139 DEGs in FB and FD, respectively, compared to CK (Supplementary Table S2). Among these DEGs, 1,543 upregulated and 1,086 downregulated genes were common to FB and FD. Pathway analysis showed that metabolism and environmental adaptation pathways were common to FB and FD, "amino sugar and nucleotide sugar metabolism" (ko00520) specific to FB and "mineral absorption" (ko04978) was specific to FD. GO enrichment analysis identified that "regulation of flower development" (GO:0009909), "cell tip growth" (GO:0009932), and "glycerol ether metabolic process" (GO:0006662) were shared by FB and FD DEGs; the top three biological processes specific to $\mathrm{FB}$ were "protein phosphorylation" (83 genes, GO:0006468), "single-organism cellular process" (72 genes, GO:0044763) and "defense response" (33 genes, GO:0006952); and "cellular metabolic process" (39 genes, GO:0044237), "cellular macromolecule metabolic process" (26 genes, GO:0044260), and "proteolysis" (20 genes, GO:0006508) were the top three biological processes specific to FD.

3.4. Gene Family Analysis. We next analyzed several gene families differentially expressed during the loranthus haustoria development and formation (Supplementary Table S2), such as ribosomal protein (RP), transcription factor (TF), ubiquitin, heat shock protein (HSP), auxin, and diseaseresistant protein (DRP) (Table 2).

3.4.1. Ribosomal Protein. Among the 2,576 RP genes, 255 were differentially expressed in the loranthus haustoria development (Figure 3(a)). In details, 7 and 5 RP genes (2 shared) were downregulated in $\mathrm{FB}$ and $\mathrm{FD}$, respectively, compared to CK. Interestingly, CK 123 and 200 RP genes were upregulated in $\mathrm{FB}$ and $\mathrm{FD}$, respectively, of which 79 were shared. Furthermore, out of the 121 upregulated RP genes exclusively in FD, 116 were downregulated in FB relative to $\mathrm{FD}$. The expression profiles of $\mathrm{RP}$ genes in $\mathrm{FB}$ and FD indicate that they might have different functions in the development and formation of loranthus haustoria.

3.4.2. Transcription Factor. We identified 863 TF genes in the loranthus haustoria, of which 174 were dysregulated in the developmental process. We found that most of the dysregulated TFs were shared (Figure 3(b)) by FB and FD. Next, we analyzed the expression changes of some TF subfamilies (Table 2, Figure 3(c)), including ethylene-responsive (ER), MYB, WRKY, and bHLH. Compared to CK, 9 ER, 5 MYB, $6 \mathrm{WRKY}$, and $11 \mathrm{bHLH}$ TF genes were upregulated in both FB and FD (Figure 3(c)). Some TF genes were specifically upregulated in FB or FD. For example, $1 \mathrm{ER}, 4 \mathrm{WRKY}$, and 5 bHLH TF genes were upregulated only in FB, while 10 $\mathrm{ER}, 5 \mathrm{WRKY}, 6 \mathrm{bHLH}$, and 4 MYB TF genes were upregulated only in FD. This indicates that these TFs might be functionally translated as required for different stages. No TF genes were upregulated along with the loranthus haustoria development; however, we found some key TF genes started their upregulation from FB, including 9 ER, 4 WRKY, and 4 MYB (Supplementary Table S2).

3.4.3. Ubiquitin. We identified 1,194 ubiquitin genes in the loranthus haustoria, of which 81 were dysregulated (Table 2, Supplementary Table S2). Among the 17 downregulated ubiquitin genes in $\mathrm{FB}$ relative to $\mathrm{CK}, 11$ were also downregulated in FD and the other 6 were increased but had no significance in FD, compared to CK. While out of the 38 upregulated ubiquitin genes in FB compared to $\mathrm{CK}, 26$ were upregulated in FD as well (Figure 3(d)). It is notable that 6 ubiquitin genes were increased along with the haustoria developmental process, including 3 polyubiquitin, 2 ubiquitin-40S RP, and 1 E3 ubiquitin-protein ligase genes.

3.4.4. Disease Resistance Protein. We assembled 226 genes encoding DRPs in the loranthus haustoria, of which 94 were dysregulated (Table 2). It is notable that most of the DRP genes were upregulated. Figure 3(e) reveals 87 (out of 94 DEGs) were upregulated in FB and FD, of which 51 were shared. We only identified 15 DEGs (7 upregulated and 8 downregulated) in FD relative to FB (Supplementary Table S2). The upregulation of ubiquitin genes (Figure 3(e)) revealed that they might be functional in the loranthus haustoria development.

3.5. miRNA Host Genes and Their Target Genes. We identified 32 miRNA host genes (Supplementary Table S1) and 4 (miR156c, miR156d, miR166d, and miR396a) were dysregulated in the process (Figure 4(a)). We next predicted the target genes for these miRNAs and found that they had no common target genes except miR156a and miR156c (Figure 4(b)). The dysregulation of miRNA host genes might explain the change of their target genes, such as TRINITY_DN3353_c2_g1, TRINITY_DN2184_c0_g1, 


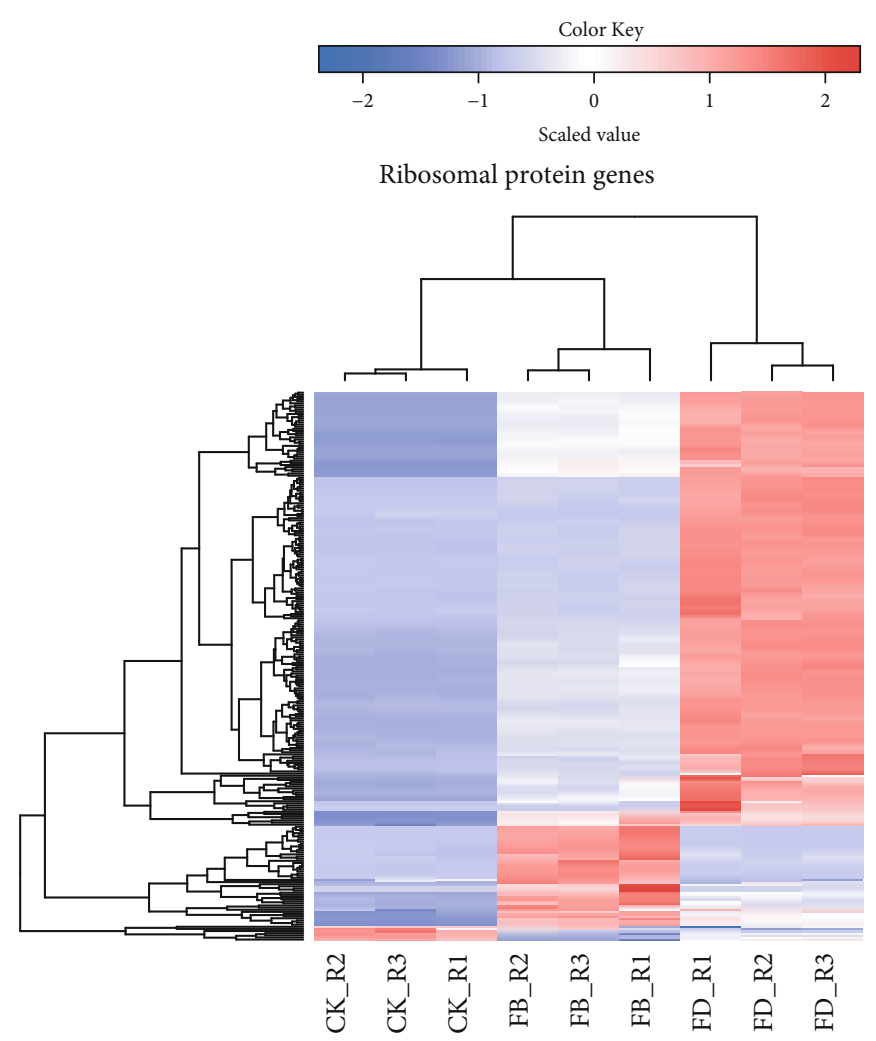

(a)

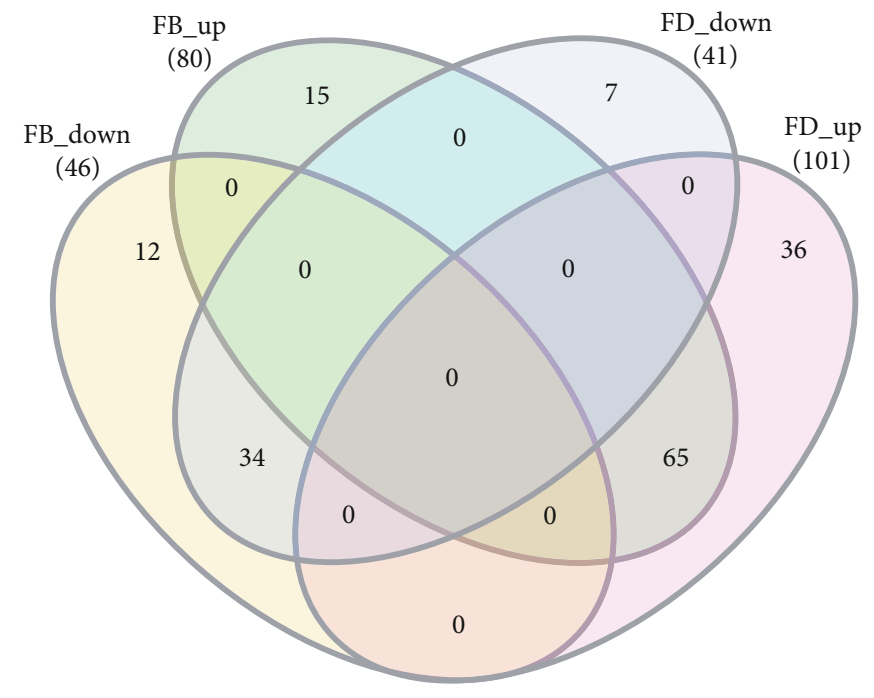

(b)

MYB

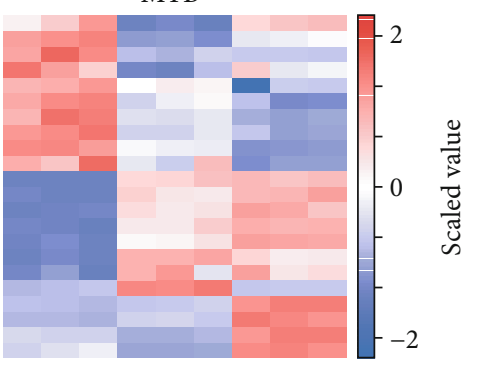

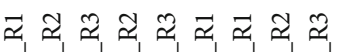

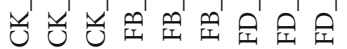
bHLH

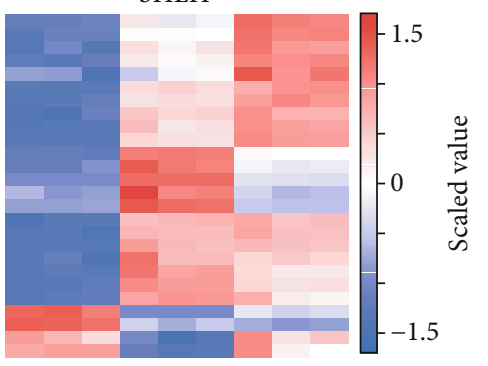

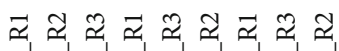

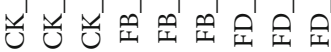

(c)

Figure 3: Continued. 


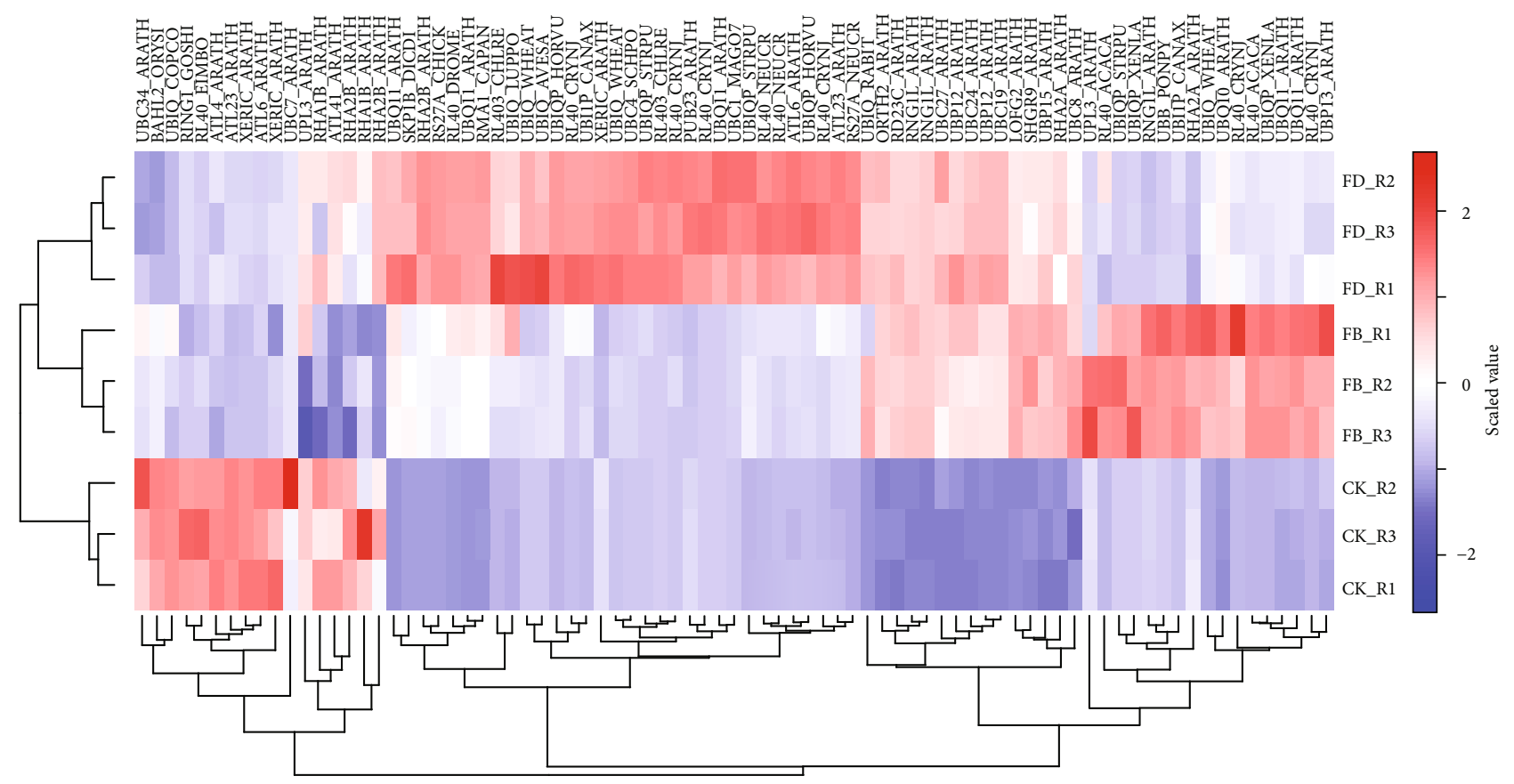

(d)

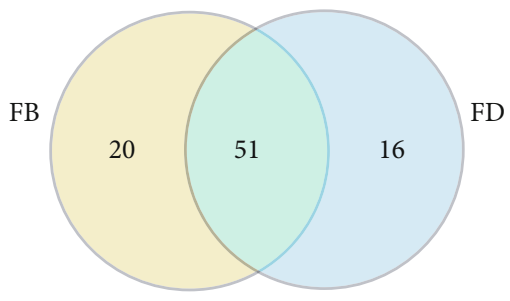

Box plot of gene expression levels of DRP

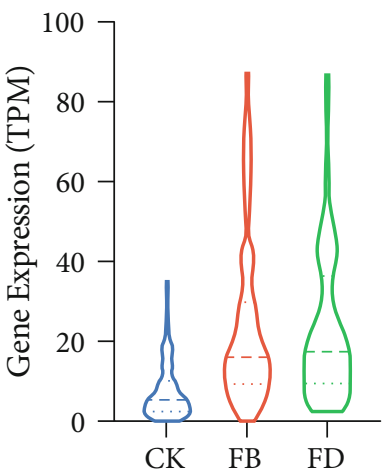

(e)

FIGURe 3: Gene family analysis of the DEGs. (a) Heat map of the RP gene expression. (b) Venn diagram of DEGs encoding TFs. (c) Heat maps of DEGs encoding different TFs. (d) Heat map of DEGs encoding ubiquitin proteins. (e) Comparison of DEGs encoding DRPs in FB and FD compared to CK.

TRINITY_DN1694_c0_g1 (squamosa promoter-bindinglike protein 2), and TRINITY_DN1166_c4_g1 (Figure 4(c)).

3.6. $q R T-P C R$ Validation. We used qRT-PCR to validate the expression changes of 9 randomly selected genes in the loranthus haustoria development, and 18S rRNA was used as internal control. The primer sequences of these genes can be found in Supplementary Table S3. The comparison of
RNA-Seq and qRT-PCR results can be found in Table 3. Overall, 22 (81.48\%) out of 27 events were agreed by both RNA-Seq and qRT-PCR. The expression patterns of 6 genes, including TRINITY_DN10066_c0_g1, TRINITY_ DN3842_c0_g2, TRINITY_DN6353_c3_g1, TRINITY_ DN6903_c0_g1, TRINITY_DN7338_c0_g1, and TRINITY_ DN759_c0_g2, were consistent in RNA-Seq and qRT-PCR. High agreement of gene expression patterns in RNA-Seq 

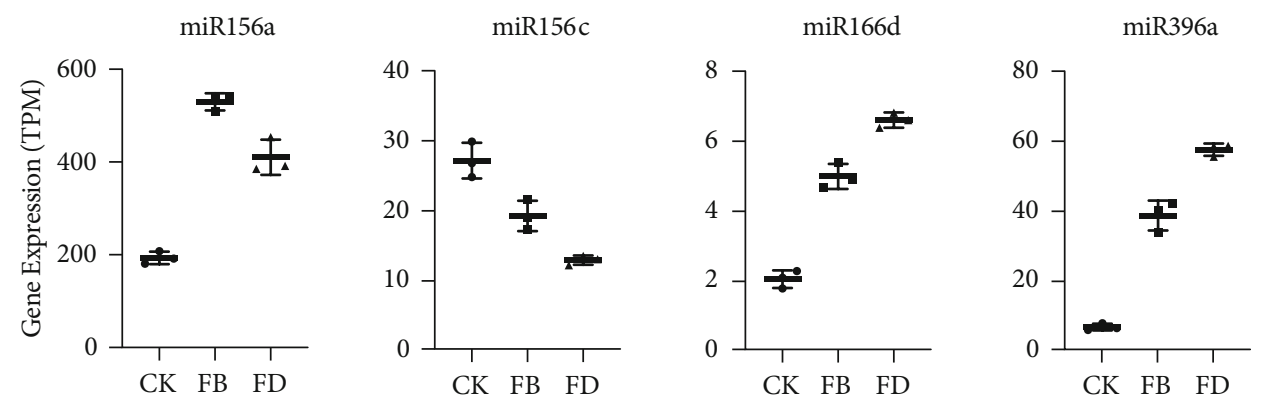

(a)

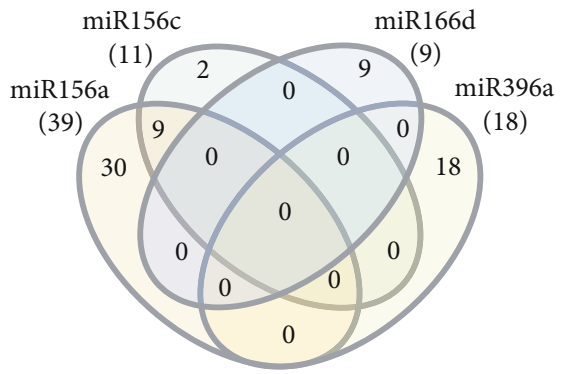

(b)
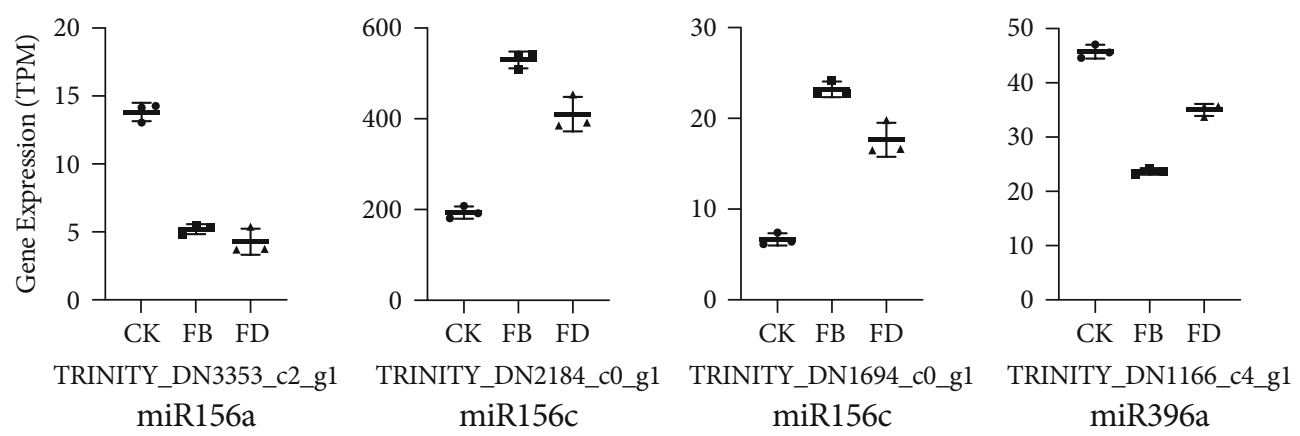

(c)

FiguRE 4: Expression of miRNA host genes and their targets. (a) Expression of host genes of miR156a, miR156c, miR166d, and miR396a. (b) Comparison of target genes for the four miRNAs. (c) Expression of target genes corresponding to miR156a, miR156c, miR166d, and miR396a.

and qRT-PCR indicates that the genes identified in this study might be functional during the loranthus haustoria development, which requires future functional experiments.

\section{Discussion}

Some studies have shown TFs' function in both parasitic plants and their hosts during the infection. For example, the upregulation of AtWRKY is important for the seeding site establishment of plant-parasitic nematodes [25]. Nearly one-half of the mobile mRNAs transferred from tomato or pumpkin to their parasitic plant Cuscuta pentagona were regulatory genes such as TFs and calmodulin proteins [26]. These evidences suggest that endogenous or exogenous TFs are important for the interaction of parasitic plants. We identified the dysregulation of bHLH, ER, MYB, and WRKY TFs (Table 2, Figure 3(c)), which may function in the formation and development of endosperm chalazal haustorium in Taxillus chinensis. These TFs have been reported to be inducible by the various environmental stresses, such as cold, drought, pathogen infection, and wounding, and be functional in the plant defense [27].

In parasitic plants, RP genes might play a key role in the survival and development. During the evolutional of Epifagus virginiana, although some RP genes are deleted, the E. virginiana plastid genomes are still transcribed and translated due to the fulfilled function by the nuclear components [28]. In addition, RPs have shown higher level of accumulation in resistant sunflower plants after the sunflower broomrape infection [29]. We found 258 out of 2,576 RP genes differentially expressed during the loranthus haustoria developmental and most are upregulated (Figure 3(a), Supplementary Table S2). We assume that both host and parasitic plants have RP genes elevated during the early phase of parasitism.

Some studies have uncovered the functions of ubiquitin proteins in the parasitism in plants and animals. For example, a unique ubiquitin carboxyl extension protein (grUBCEP12) is secreted by the plant-parasitic nematode 


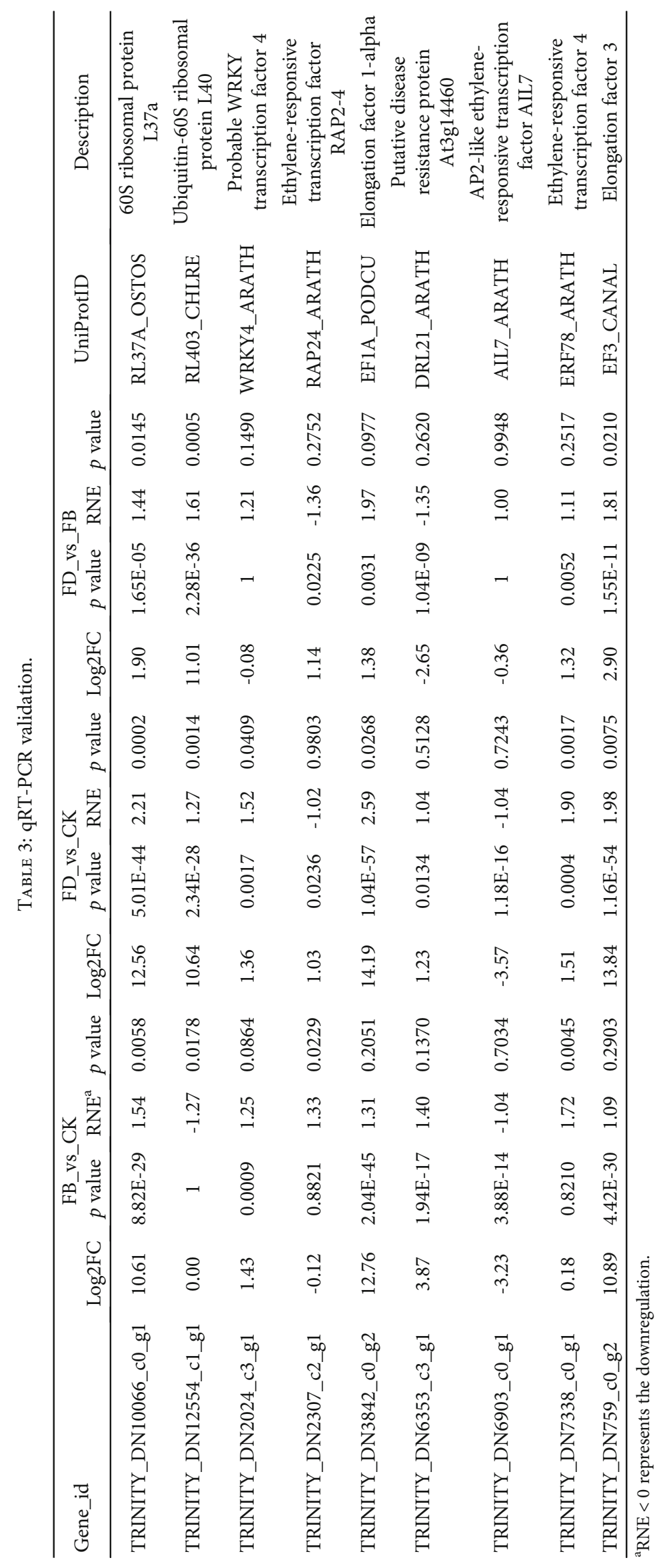


Globodera rostochiensis can promote successful plant parasitism through suppressing the plant's defense through the suppression of plant immunity and can further generate within root tissue the feeding cells essential for nematode development [30]. Rhiannon reported that E2 and E3 ubiquitin proteins secreted by the parasitic nematode Trichinella spiralis have the capacity of modifying the host skeletal muscle cells [31]. In this study, we identified 66, 176, and 540 genes encoding E1, E2, and E3 ubiquitin enzymes, respectively. Among them, 81 were differentially expressed (Table 2, Figure 3(d)), including 8 E2 and 29 E3 ubiquitin genes (Supplementary Table S2). Based on these evidences, we assume that the secretion of ubiquitin genes and proteins by loranthus has positive efforts in the parasitism. While further experiments are required to study the functions of ubiquitin genes and proteins in the parasitism of loranthus.

A recent study reported that Arabidopsis thaliana mRNAs are targeted by miRNAs produced by Cuscuta campestris during the parasitism, resulting in mRNA cleavage, secondary siRNA production, and decreased mRNA accumulation [11]. Here, we predicted 32 miRNA host genes in the loranthus haustoria (Supplementary Table S1) and identified the dysregulation of miR156c, miR156d, miR166d, and miR396a (Figure 4). Due to the limited information of loranthus genes, we only found a few genes targeted by these four miRNAs (Figure 4). Further experiments are required to identify the mature miRNA sequences and their function in the loranthus haustoria development.

\section{Conclusions}

In conclusion, we studied the transcriptome profiles of the loranthus haustoria development. We assembled 160,571 loranthus genes and annotated them by aligning them to NR, GO, KEGG, UniProt/Swiss-Prot, Pfam, and EggNOG databases. After lowly expressed genes were filtered, we identified 18,360 genes in the loranthus haustoria, of which 3,749 and 4,139 were dysregulated in FB and FD, respectively, compared to CK. Some important gene families were found to be related to the loranthus haustoria development, such as transcription factor, ubiquitin, ribosomal protein, and diseaseresistant protein. Further, 32 miRNA host genes were identified and the dysregulation of 4 miRNA host genes might be one of the reasons for some genes which are dysregulated as well in the process. This is the first time to report the transcriptome of loranthus haustoria. It will provide valuable resources to other studies. More importantly, the findings of this study will improve our understanding of parasitism and contribute to the breeding program of loranthus.

\section{Data Availability}

The raw sequencing data can be accessed from the NCBI Sequence Read Archive (SRA) platform (https://trace.ncbi .nlm.nih.gov/Traces/sra/) under the accession number SRA896707. The assembled transcriptome of loranthus haustoria can be accessed in the TSA database of NCBI under the accession number GHNL00000000.

\section{Conflicts of Interest}

The authors declare that there is no conflict of interest.

\section{Authors' Contributions}

SW, JF, and LP conceived and designed the experiments. SW, $\mathrm{LH}, \mathrm{HL}$, and XJ performed the experiments. SW, YW, and LW analyzed the data. SW and JF wrote the manuscript. LP revised the manuscript. All the authors have read and approved the final version of manuscript.

\section{Acknowledgments}

This project was supported by the National Science Foundation of China [81660637, 81703649, and 81860672]; Guangxi National Science Foundation [2016GXNSFDA380012, 2017GXNSFDA198026, and 2018GXNSFAA281089]; and Guangxi Botanical Garden of Medicinal Plants Research and Innovation Team Building Project [GYCH2019008]. We acknowledge Mister Dajie Zhou from BGI-Shenzhen for his help with the deep sequencing.

\section{Supplementary Materials}

Supplementary 1. Table S1. miRNA host genes and sequences.

Supplementary 2. Table S2: dysregulated genes identified in $\mathrm{FB}$ and FD compared to CK.

Supplementary 3. Table S3: forward and reverse primers used in the qRT-PCR experiment.

\section{References}

[1] S. Wei, X. Ma, L. Pan et al., "Transcriptome analysis of Taxillusi chinensis (DC.) Danser seeds in response to water loss," PLoS One, vol. 12, no. 1, article e0169177, 2017.

[2] C. Y. Liu, Y. C. Lin, J. S. Deng, J. C. Liao, W. H. Peng, and G. J. Huang, "Antioxidant, anti-inflammatory, and antiproliferative activities of Taxillus sutchuenensis," The American Journal of Chinese Medicine, vol. 40, no. 2, pp. 335-348, 2012.

[3] S. Yoshida, S. Cui, Y. Ichihashi, and K. Shirasu, "The Haustorium, a specialized invasive organ in parasitic plants," Annual Review of Plant Biology, vol. 67, no. 1, pp. 643-667, 2016.

[4] X. Zhang, O. Berkowitz, J. A. Teixeira da Silva et al., "RNA-Seq analysis identifies key genes associated with haustorial development in the root hemiparasite Santalum album," Frontiers in Plant Science, vol. 6, p. 661, 2015.

[5] P. C. Bandaranayake, T. Filappova, A. Tomilov et al., "A single-electron reducing quinone oxidoreductase is necessary to induce haustorium development in the root parasitic plant Triphysaria," Plant Cell, vol. 22, no. 4, pp. 1404-1419, 2010.

[6] X. Zhang, J. A. Teixeira da Silva, J. Duan, R. Deng, X. Xu, and G. Ma, "Endogenous hormone levels and anatomical characters of haustoria in Santalum album L. seedlings before and after attachment to the host," Journal of Plant Physiology, vol. 169, no. 9, pp. 859-866, 2012.

[7] T. D. Nikam and R. B. Barmukh, "GA3 enhances in vitro seed germination in Santalum album," Seed Science and Technology, vol. 37, no. 2, pp. 276-280, 2009. 
[8] D. Barrett and J. E. Fox, "Santalum album: kernel composition, morphological and nutrient characteristics of pre-parasitic seedlings under various nutrient regimes," Annals of Botany, vol. 79, no. 1, pp. 59-66, 1997.

[9] A. Ranjan, Y. Ichihashi, M. Farhi et al., "De novo assembly and characterization of the transcriptome of the parasitic weed dodder identifies genes associated with plant parasitism," Plant Physiology, vol. 166, no. 3, pp. 1186-1199, 2014.

[10] R. Weßling, S. M. Schmidt, C. O. Micali et al., "Transcriptome analysis of enriched Golovinomyces orontii haustoria by deep 454 pyrosequencing," Fungal Genetics and Biology, vol. 49, no. 6, pp. 470-482, 2012.

[11] S. Shahid, G. Kim, N. R. Johnson et al., "MicroRNAs from the parasitic plant Cuscuta campestris target host messenger RNAs," Nature, vol. 553, no. 7686, pp. 82-85, 2018.

[12] M. Chen, R. Xu, A. Rai et al., "Distinct shed microvesicle and exosome microRNA signatures reveal diagnostic markers for colorectal cancer," PLoS One, vol. 14, no. 1, article e0210003, 2019.

[13] M. Chen, R. Xu, H. Ji et al., "Transcriptome and long noncoding RNA sequencing of three extracellular vesicle subtypes released from the human colon cancer LIM1863 cell line," Scientific Reports, vol. 6, no. 1, article 38397, 2016.

[14] R. D. Finn, J. Clements, and S. R. Eddy, "HMMER web server: interactive sequence similarity searching," Nucleic Acids Research, vol. 39, Supplement 2, pp. W29-W37, 2011.

[15] T. N. Petersen, S. Brunak, G. von Heijne, and H. Nielsen, "SignalP 4.0: discriminating signal peptides from transmembrane regions," Nature Methods, vol. 8, no. 10, pp. 785-786, 2011.

[16] A. Krogh, B. Larsson, G. von Heijne, and E. L. L. Sonnhammer, "Predicting transmembrane protein topology with a hidden markov model: application to complete genomes ", Journal of Molecular Biology, vol. 305, no. 3, pp. 567-580, 2001.

[17] K. Lagesen, P. Hallin, E. A. Rødland, H. H. Staerfeldt, T. Rognes, and D. W. Ussery, "RNAmmer: consistent and rapid annotation of ribosomal RNA genes," Nucleic Acids Research, vol. 35, no. 9, pp. 3100-3108, 2007.

[18] S. Powell, K. Forslund, D. Szklarczyk et al., "eggNOG v4.0: nested orthology inference across 3686 organisms," Nucleic Acids Research, vol. 42, no. D1, pp. D231-D239, 2014.

[19] A. Conesa, S. Götz, J. M. García-Gómez, J. Terol, M. Talón, and M. Robles, "Blast2GO: a universal tool for annotation, visualization and analysis in functional genomics research," Bioinformatics, vol. 21, no. 18, pp. 3674-3676, 2005.

[20] L. Kong, Y. Zhang, Z. Q. Ye et al., "CPC: assess the proteincoding potential of transcripts using sequence features and support vector machine," Nucleic Acids Research, vol. 35, Supplement 2, pp. W345-W349, 2007.

[21] R. Li, C. Yu, Y. Li et al., "SOAP2: an improved ultrafast tool for short read alignment," Bioinformatics, vol. 25, no. 15, pp. 1966-1967, 2009.

[22] H. J. Wu, Y. K. Ma, T. Chen, M. Wang, and X. J. Wang, "PsRobot: a web-based plant small RNA meta-analysis toolbox," Nucleic Acids Research, vol. 40, no. W1, pp. W22-W28, 2012.

[23] B. Li and C. N. Dewey, "RSEM: accurate transcript quantification from RNA-Seq data with or without a reference genome," BMC Bioinformatics, vol. 12, p. 323, 2011.

[24] M. D. Robinson, D. J. McCarthy, and G. K. Smyth, "edgeR: a bioconductor package for differential expression analysis of digital gene expression data," Bioinformatics, vol. 26, no. 1, pp. 139-140, 2010.
[25] W. Grunewald, M. Karimi, K. Wieczorek et al., "A role for AtWRKY23 in feeding site establishment of plant-parasitic nematodes," Plant Physiology, vol. 148, no. 1, pp. 358-368, 2008.

[26] J. H. Westwood, J. K. Roney, P. A. Khatibi, and V. K. Stromberg, "RNA translocation between parasitic plants and their hosts," Pest Management Science, vol. 65, no. 5, pp. 533-539, 2009.

[27] K. Singh, R. C. Foley, and L. Onate-Sanchez, "Transcription factors in plant defense and stress responses," Current Opinion in Plant Biology, vol. 5, no. 5, pp. 430-436, 2002.

[28] J. I. Yoder, "Parasitic plant responses to host plant signals: a model for subterranean plant-plant interactions," Current Opinion in Plant Biology, vol. 2, no. 1, pp. 65-70, 1999.

[29] C. Yang, L. Xu, N. Zhang et al., "iTRAQ-based proteomics of sunflower cultivars differing in resistance to parasitic weed Orobanche cumana," Proteomics, vol. 17, no. 13-14, 2017.

[30] S. Chen, D. Chronis, and X. Wang, “The novel GrCEP12 peptide from the plant-parasitic nematode Globodera rostochiensis suppresses flg22-mediated PTI," Plant Signaling \& Behavior, vol. 8, no. 9, article e25359, 2013.

[31] R. R. White, A. H. Ponsford, M. P. Weekes et al., "Ubiquitindependent modification of skeletal muscle by the parasitic nematode, Trichinella spiralis," PLoS Pathogens, vol. 12, no. 11, article e1005977, 2016. 